\title{
INVARIANTS FOR REDUCIBLE SYSTEMS OF MEROMORPHIC DIFFERENTIAL EQUATIONS
}

\author{
by W. BALSER*, W. B. JURKAT $\dagger$ and D. A. LUTZ† \\ (Received 12th October 1978)
}

\section{Introduction}

With differential equations in the neighbourhood of an irregular singular point, it sometimes happens that formal solutions may converge. For example, this occurs for Bessel's equation at $\infty$ when the parameter is half of an odd integer. In addition, there are some classical theorems of Perron and Lettenmeyer which give sufficient conditions for the existence of linearly independent analytic solutions at (generally) an irregular singular point. Using the principle of reduction of order, such a solution may be used to transform the differential equation into one whose coefficient matrix is triangularly blocked with an $(n-1)$ and 1-block on the diagonal. The solutions of the given differential equation can thus be obtained by solving a lower dimensional differential equation plus quadrature.

More generally, one may consider a meromorphic differential equation [A] which is equivalent to $[\mathrm{B}]$ whose cofficient matrix is of the form

$$
B(z)=\left[\begin{array}{ll}
B_{11}(z) & 0 \\
B_{21}(z) & B_{22}(z)
\end{array}\right]
$$

with square diagonal blocks of dimension at least one. Such a differential equation $[\mathrm{A}]$ is called reducible.

It was shown in (3) (in the distinct eigenvalue case) and later in (6) (without restriction) that there exist for [B] a formal fundamental solution, corresponding normal solutions, and normal connection matrices which are all blocked triangularly in the same way. This allows one to characterise reducibility in terms of conditions involving the block structure of the invariants.

In an example like (0.1) one can say, roughly speaking, that the zeros in the coefficient matrix appear also in the solutions of [A]. Hence one may ask how generally this can hold. If we take, for example, a system [A] corresponding to the $n$th order equation $y^{(n)}-y=0$, then

$$
A(z)=\left[\begin{array}{ccccc}
0 & 1 & 0 & . & 0 \\
\cdot & \cdot & \cdot & & \\
\cdot & & \cdot & . & \\
\cdot & & & \cdot & 1 \\
1 & . & . & \cdot & 0
\end{array}\right]
$$

* Contributions made while being a Visiting Associate Professor at the University of Wisconsin-Milwaukee. † Supported in part by grants from the National Science Foundation. 
has a large number of zeros, but every fundamental solution matrix can be seen to have all non-zero elements. Therefore we cannot expect every zero position of $A$ to imply a zero in the corresponding position of some fundamental solution. However, if we require that the non-zero positions of $A(z)$ are contained in a transitive position set $\sigma$ (see Section 1 ), then the iteration process of the Picard-Lindelof existence theorem, starting with the $I$ matrix, could be used to obtain an actual fundamental solution differing from $I$ only in positions contained in $\sigma$. For formal solutions, however, this argument does not apply. Nevertheless we prove in Section 1 the existence of a formal fundamental solution $H$ of [A] having the same property, and we furthermore show that the normal solutions and consequently all the invariants corresponding to $(A, H)$ have the same structure.

Section 2 characterises reducible equations, i.e. equations which are meromorphically resp. analytically resp. root-meromorphically equivalent to a system [A] as described above, in terms of their invariants. This allows to check simply by investigation of the invariants how far a system can be simplified by means of meromorphic resp. analytic resp. root-meromorphic transformations. Again, this section extends the results of $(3),(6)$ for the case of lower triangular reducibility.

As an application of the discussions in Sections 1 and 2 we characterise in Section 3 all formal vector solutions $h(z)$ of [A], which in a natural sense can be said to converge, in terms of the invariants and show how they can be explicitly found and used to reduce the size of the system. Since a special case of a convergent formal vector solution is a solution which is analytic at $\infty$, our results in particular characterise the existence of such solutions in terms of the invariants. Recently, in determining the index of the differential operator corresponding to an $n$th order scalar meromorphic differential equation, B. Malgrange (8) has given sufficient conditions for the existence of analytic solutions, which may be thought to generalise the classical Perron and Lettenmeyer theorems. L. M. Hall (5) has recently given some necessary and sufficient conditions for the same question. They are generally difficult to apply but in light of the above mentioned correspondence between convergent formal solutions and invariants, these conditions are equivalent to the vanishing of certain invariants.

In the case of reduced differential equations whose diagonal blocks have known normal solutions; one may find the normal solutions of the full differential equation and calculate the corresponding invariants explicitly (Section 4). Section 5 covers the "inverse" problem, namely an explicit construction of a reduced differential equation having given invariants, provided that the same problem for the diagonal blocks is already solved. In a final Section 6, we consider certain optimal types of reducibility.

For the notations used in this paper, see (1) and (2).

\section{Reduced Systems}

Let $\sigma$ be an arbitrary transitive set of positions $(j, k), 1 \leqq j, k \leqq n$. We call a $n \times n$-matrix reduced of type $\sigma$, if its elements are non-zero only for positions in $\sigma$. Using the transitivity of $\sigma$, we see that the set of matrices reduced of type $\sigma$ is closed under matrix multiplication.

Given a position set $\sigma$, there exists a uniquely defined matrix with ones in all positions included in $\sigma$ and zeros elsewhere. We call this matrix the incidence matrix and denote it also by $\sigma$. Given a transitive set $\sigma$, then a permutation $\pi$ of $\{1, \ldots, n\}$ yields an equivalent 
transitive set, say $\tilde{\sigma}$, and the corresponding incidence matrices are related by

$$
\tilde{\sigma}=R^{-1} \sigma R,
$$

where $R$ is a permutation matrix determined by $\pi$. We first want to show that we can choose $R$ in a way that $\tilde{\sigma}$ shows a lower triangular block structure.

Lemma 1. Let $\sigma$ be an incidence matrix corresponding to an arbitrary transitive position set. Then there exists an equivalent set $\tilde{\sigma}$ whose incidence matrix is lower triangularly blocked in such a way that a position in a block shows a one iff every position in the same block does, and that the position indices of the off-diagonal non-zero blocks form a transitive and antisymmetric set.

Proof. We define for $1 \leqq j, k \leqq n$

$$
j \sim k \text { iff } j=k \text { or }(j, k) \text { and }(k, j) \in \sigma .
$$

This is an equivalence relation on $\{1, \ldots, n\}$, and by a suitable permutation we can arrange that the equivalence classes consist of consecutive numbers. This permutation takes $\sigma$ into $\hat{\sigma}=\hat{R}^{-1} \sigma \hat{R}$ which shows a block structure such that all the positions in a block are equal, and that the indices of the non-zero off-diagonal blocks form a transitive and antisymmetric set. If $\sigma=\{1, \ldots, n\}^{2}$, i.e. the set of all pairs $(j, k)(1 \leqq j, k \leqq n)$, then there is only one (diagonal) block of all ones, and we call $\sigma$ trivial. For a non-trivial $\sigma$ this transitive and antisymmetric set is non-empty. Then applying a second permutation we can arrange that $\tilde{\sigma}=\tilde{R}^{-1} \hat{\sigma} \tilde{R}$ is lower triangularly blocked (see $(2$, Section 3$)$ ). This completes the proof.

We call every transitive position set $\sigma$ for which we can take $R=I$ in Lemma 1 a normalised transitive set. The normalised sets are the most interesting ones for the following discussion, however we wish to consider others, too. For a given transitive $\sigma$, we call a matrix reduced of type $\sigma^{+}$if it is the sum of the identity matrix and a matrix which is reduced of type $\sigma$. For normalised $\sigma$, this means that a matrix is reduced of type $\sigma^{+}$iff it is reduced of type $\sigma$ except that it has $I$-blocks on the diagonal where $\sigma$ has zero-blocks.

Proposition 1. Let $[A]$ be a meromorphic differential equation, where $A(z)$ is reduced of type $\sigma$. Then there exists a formal fundamental solution matrix $H(z)=\Psi(z) \exp [Q(z)]$ (with in general not normalised $Q(z)$ ) such that $H$ is reduced of type $\sigma^{+}$.

Furthermore, for every such $H(z)$ and every integer $\nu$ there exists an actual fundamental solution matrix $Y_{\nu}(z)$ which is also reduced of type $\sigma^{+}$and satisfies

$$
Y_{\nu}(z) \cong H(z) \text { in } S_{\nu}
$$

Remark. The set of matrices reduced of type $\sigma^{+}$is also closed under matrix multiplication and the set of all invertible matrices reduced of type $\sigma^{+}$forms a group. Hence, if $H=\Psi e^{O}$ is reduced of type $\sigma^{+}$, then the formal circuit matrix $e^{2 \pi i L}$ is reduced of type $\sigma^{+}$, too. Therefore we may choose $L$ to be also reduced of type $\sigma$ (see (4, p. 104)). Since $e^{-O}$ and $z^{-L}$ are reduced of type $\sigma^{+}$, we have

$$
H=F_{m} z^{L} e^{Q},
$$

where $F_{m}$ is a formal meromorphic transformation and also reduced of type $\sigma^{+}$. 
An inspection of the proof of $(\mathbf{1}$, Lemma 2$)$ shows that every formal meromorphic transformation $F_{m}$ which is reduced of type $\sigma^{+}$for a normalised $\sigma$ can be uniquely factored as

$$
F_{m}=F_{a} P(z) z^{\kappa}
$$

where $P(z)$ is a lower triangular matrix polynomial with $\operatorname{diag} P(z)=I$ and $P(o)=I, K$ is a diagonal matrix of integers, and $P(z) z^{K}$ is reduced of type $\sigma^{+}$.

Proof. For the proof we may without loss of generality assume that $\sigma$ is a normalised transitive set, because the general case follows by applying the same permutation similarity transformation to all the matrices involved. For such a $\sigma$, we proceed by induction over $n$, and we may assume $\sigma$ to be non-trivial.

If $n=1$, the proposition is trivial. If $n>1$, we partition

$$
A(z)=\left[\begin{array}{ll}
A_{11}(z) & A_{12}(z) \\
A_{21}(z) & A_{22}(z)
\end{array}\right],
$$

where $A_{j j}$ is of dimension $n_{j} \times n_{j}, n_{1}+n_{2}=n$, and $1 \leqq n_{1} \leqq n-1$ is chosen so that it corresponds to the lower triangular block structure of $\sigma$. Then we see that $A(z)$ being reduced of type $\sigma$ implies $A_{12}(z) \equiv 0$ and $A_{j j}$ reduced of type $\sigma_{j}$, where

$$
\begin{aligned}
& \sigma_{1}=\sigma \cap\left\{1, \ldots, n_{1}\right\}^{2}, \\
& \sigma_{2}=\sigma \cap\left\{n_{1}+1, \ldots, n\right\}^{2} .
\end{aligned}
$$

For $j=1,2$, the set $\sigma_{j}$ is again transitive and normalised, and by induction hypothesis we see that $\left[A_{j j}\right]$ has a formal fundamental solution matrix $H_{j j}=\Psi_{j j} e^{Q} j$, such that $H_{j j}$ is reduced of type $\sigma_{i}^{+}$.

If we define

$$
H_{21}(z)=H_{22}(z) \int H_{22}^{-1}(z) A_{21}(z) H_{11}(z) d z=\Psi_{21} e^{Q_{1}}
$$

(for the definition and existence of the formal integral see $(6, p .132)$ ), then

$$
H(z)=\left[\begin{array}{ll}
H_{11} & 0 \\
H_{21} & H_{22}
\end{array}\right]
$$

is a formal fundamental solution of [A]. In order to show that $H$ is reduced of type $\sigma^{+}$, we rewrite (1.4) as

$\left[\begin{array}{ll}0 & 0 \\ H_{21}(z) & 0\end{array}\right]=\left[\begin{array}{lr}0 & 0 \\ 0 & H_{22}(z)\end{array}\right] \int\left[\begin{array}{lr}0 & 0 \\ 0 & H_{22}^{-1}(z)\end{array}\right]\left[\begin{array}{ll}0 & 0 \\ A_{21}(z) & 0\end{array}\right]\left[\begin{array}{ll}H_{11}(z) & 0 \\ 0 & 0\end{array}\right] d z$.

Here the integrand is reduced of type $\sigma$ and so is the left side, since the formal integral assigns to the zero-expression the zero-expression as an antiderivative (see (6)).

To obtain the existence of a reduced actual solution for any reduced formal solution and for arbitrary $\nu$, let the matrix $Y_{\nu, j j}$ be an actual fundamental solution of $\left[A_{j j}\right]$ satisfying

$$
Y_{\nu, j j} \cong H_{j j} \text { in } S_{\nu}
$$

which is reduced of type $\sigma_{j}^{+}$(the existence following by the induction hypothesis).

The same argument as in the formal case shows that for 
the matrix

$$
Y_{\nu, 21}(z)=Y_{\nu, 22}(z) \int Y_{\nu, 22}^{-1}(z) A_{21}(z) Y_{\nu, 11}(z) d z
$$

$$
Y_{\nu}=\left[\begin{array}{ll}
Y_{\nu, 11} & 0 \\
Y_{\nu, 21} & Y_{\nu, 22}
\end{array}\right]
$$

is a fundamental solution of [A] which is reduced of type $\sigma^{+}$, provided we define the integral to mean an antiderivative considered on the Riemann surface of $\log z$, and the antiderivative of the zero function is chosen to be the zero function. This $Y_{\nu}$ may not satisfy (1.3), however there exists a constant invertible matrix

$$
C=\left[\begin{array}{ll}
C_{11} & C_{12} \\
C_{21} & C_{22}
\end{array}\right]
$$

such that

$$
Y_{\nu} C=[\Psi]_{S_{\nu}} e^{Q}
$$

Calculating the product on the left-hand side, we see

$$
Y_{\nu, 11} C_{11}=\left[\Psi_{11}\right]_{s_{\nu}} e^{Q_{1}}, \quad Y_{\nu, 11} C_{12}=[0]_{S_{\nu}} e^{Q_{2}} .
$$

We also have $Y_{\nu, 11}=\left[\Psi_{11}\right]_{S_{\nu}} e^{Q_{1}}$, therefore

$$
e^{Q_{1}} C_{11} e^{-Q_{1}} \cong I, \quad e^{Q_{1}} C_{12} e^{-Q_{2}} \cong 0 \text { in } S_{v} .
$$

This shows that

$$
\left[\begin{array}{cc}
C_{11}^{-1} & -C_{11}^{-1} C_{12} \\
0 & I
\end{array}\right]
$$

is a transition matrix in $S_{v}$, hence for

$$
\tilde{C}=C\left[\begin{array}{cc}
C_{11}^{-1} & -C_{11}^{-1} C_{12} \\
0 & I
\end{array}\right]=\left[\begin{array}{cc}
I & 0 \\
\tilde{C}_{21} & \tilde{C}_{22}
\end{array}\right]
$$

we have

$$
Y_{\nu} \tilde{C}=[\Psi]_{S_{\nu}} e^{O} \text {, in particular, } Y_{\nu, 22} \tilde{C}_{22}=\left[\Psi_{22}\right]_{S_{\nu}} e^{O_{2}}
$$

Utilising $Y_{\nu, 22}=\left[\Psi_{22}\right] e^{O_{2}}$, we see that

$$
\left[\begin{array}{cc}
I & 0 \\
0 & \tilde{C}_{22}
\end{array}\right]
$$

is also a transition matrix in $S_{\nu}$, hence

$$
Y_{\nu}\left[\begin{array}{cc}
I & 0 \\
\hat{C}_{21} & I
\end{array}\right]=[\Psi]_{S_{\nu}} e^{Q}
$$

To complete the proof, we expand the product $Y_{\nu} \hat{C}$, and see that the $(2,1)$ position is

$$
Y_{\nu, 21}+Y_{\nu, 22} \hat{C}_{21}=\left[\Psi_{21}\right]_{S_{\nu}} e^{Q_{1}} \text {. }
$$

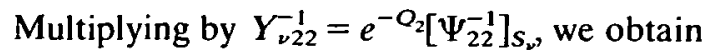

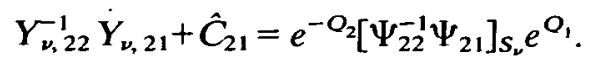


The transitivity of $\sigma$ implies that $\Psi_{22}^{-1} \Psi_{21}$ and $Y_{\nu, 22}^{-1} Y_{\nu, 21}$ both have zeros in positions not in $\sigma$. Therefore, if $\hat{C}_{21}=C_{21}(1)+C_{21}(2), C_{21}(1)$ having non-zero elements only in $\sigma, C_{21}(2)$ having non-zero elements only in positions not in $\sigma$, we see

This shows

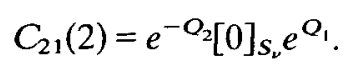

$$
\hat{C}=\left[\begin{array}{cc}
I & 0 \\
C_{21}(1) & I
\end{array}\right]\left[\begin{array}{cc}
I & 0 \\
C_{21}(2) & I
\end{array}\right]=C_{1} C_{2},
$$

where $C_{1}$ is reduced of type $\sigma^{+}$and $C_{2}$ is a transition matrix for $S_{\nu}$. Hence

$$
Y_{\nu} C_{1} \cong H \text { in } S_{\nu}
$$

and $Y_{\nu} C_{1}$ is reduced of type $\sigma^{+}$, which completes the proof.

We next aim to prove that to a given pair $(A, H)$, where both $A(z)$ and $H(z)=$ $\Psi(z) e^{Q(z)}$ are reduced of type $\sigma$ resp. $\sigma^{+}$, there correspond normal solutions and normalised connection matrices being reduced of type $\sigma^{+}$for all $\nu$. To that extent, we first state

Proposition 2. Let $[A]$ be a meromorphic differential equation and $H=\Psi e^{Q}$ a formal fundamental solution of $[A]$. For every $\nu$, assume the existence of fundamental solutions $Y_{\nu}$ such that

$$
Y_{\nu} \cong H \text { in } S_{\nu}
$$

and that the connection matrices $W_{\nu}=Y_{\nu}^{-1} Y_{\nu-1}$ are reduced of type $\sigma^{+}$for some transitive position set $\sigma$. Then the normalised connection matrices $V_{\nu}$ corresponding to $H$ are also reduced of type $\sigma^{+}$for every $\nu$. Furthermore, if $Y_{\nu}$ are reduced of type $\sigma^{+}$for all $\nu$, then the same holds for the normal solutions $X_{\nu}$.

Note that when $Q(z)=q(z) I$, the proposition follows trivially. Otherwise, for a proof when $Q$ is normalised, we refer to (6), and the generalisation to arbitrary $Q$ follows from the discussion at the end of Section 7 in (2).

Propositions 1 and 2 have the following

Corollary. Let $[A]$ be a meromorphic differential equation where $A$ is reduced of type $\sigma$ for some transitive position set $\sigma$ and let $H=\Psi e^{Q}$ be a formal fundamental solution matrix which is reduced of type $\sigma^{+}$. Then for every $\nu$ the normalised connection matrix $V_{\nu}$ and the normal solution $X_{\nu}$ are reduced of type $\sigma^{+}$. Furthermore, if $\sigma$ is normalised and $\left[A_{j j}\right]$ is a diagonal block of $[A]$ in the block structure induced by $\sigma$, then for every $\nu$ for which $\tau_{\nu}$ is a Stokes' ray for $\left[A_{j j}\right]$ the diagonal block $X_{\nu, j j}$ of $X_{\nu}$ is the normal solution corresponding to $\left(A_{j j}, H_{j j}\right)$ and the Stokes' ray $\tau_{\nu}$, and $V_{\nu, j i}$ is the corresponding normalised connection matrix. If $\tau_{v}$ is not a Stokes' ray of $\left[A_{i j}\right]$, then $V_{\nu, j i}=I$ and there are two alternatives: Either $\left[A_{i j}\right]$ has no Stokes' rays in which case $X_{\nu, j j}=H_{j j}$ (hence $H_{j j}$ is convergent) or $X_{\nu, j j}$ is the normal solution corresponding to $\left(A_{j j}, H_{j j}\right)$ and the Stokes' ray of $\left[A_{j j}\right]$ which follows $\tau_{\nu}$.

The parts of the Corollary which are not immediate consequences of the preceding Propositions, are obtained by an easy generalisation of the corresponding Korollar in (6), where a proof is given for a special case of triangular blocking.

A discussion of the off-diagonal blocks of the normal solutions and the connection system can be found in Section 4. 


\section{Reducibility}

Let $[A]$ be a meromorphic differential equation. We call $[A]$ meromorphically reducible if it is meromorphically equivalent to an equation $[\bar{A}]$ which is reduced of type $\sigma$ for some transitive set $\sigma \neq\{1, \ldots, n\}^{2}$. In this case we call $\sigma$ the type of reducibility of $[A]$. We will characterise reducible systems in terms of their invariants. To do this, we now formulate

Proposition 3. Let $\sigma$ be a transitive position set, and let $F(z)$ be a formal meromorphic transformation. Furthermore, let $T_{\nu}(z)$ be analytic matrices for $z \in S_{\nu}, z \mid>a$ (for some given $a \geqq 0$ ) and satisfying for some real numbers $0 \leqq \tau_{0}<\ldots<\tau_{m-1}<2 \pi, \tau_{m}=\tau_{0}+2 \pi$, $\tau_{m+1}=\tau_{1}+2 \pi, \tau_{-1}=\tau_{m-1}-2 \pi$,

$$
\begin{gathered}
T_{\nu}(z) \cong F(z) \quad \text { in } \quad S_{\nu}=S\left(\tau_{\nu-1}, \tau_{\nu+1}\right) \quad \text { for } \quad \nu=0, \ldots, m, \\
T_{m}(z)=T_{0}\left(z e^{-2 \pi i}\right) \text { in } S_{m-}
\end{gathered}
$$

If $T_{\nu, \nu-1}(z)=T_{\nu}^{-1}(z) T_{\nu-1}(z)$ are reduced of type $\sigma^{+}$for $\nu=1, \ldots, m$, then there exists an actual meromorphic transformation $T(z)$ such that

where $\tilde{T}_{\nu}(z)$ are reduced of type $\sigma^{+}$.

$$
T_{\nu}(z)=T(z) \tilde{T}_{\nu}(z)
$$

Proof. If $\sigma$ is trivial or the empty set, then the proposition holds, since in the first case we can take $T(z)=I$, and in the second case $T_{\nu, \nu-1}(z) \equiv I$, hence $T(z)=T_{\nu}(z)$ is independent of $\nu$ and $(2.1),(2.2)$ imply that $T(z)$ is a meromorphic transformation. Now assume $\sigma$ to be non-trivial and proceed by induction on $n$. When $n=1$, either $\sigma$ is empty or trivial. Therefore we may assume $n>1$ and also, without loss of generality, $\sigma$ to be normalised. Then take any partitioning of the incidence matrix $\sigma$ of the form

$$
\sigma=\left[\begin{array}{cc}
\sigma_{1} & 0 \\
\sigma_{21} & \sigma_{2}
\end{array}\right]
$$

$\sigma_{i}$ of dimension $n_{i} x n_{i}, i=1,2$, where $n_{i} \geqq 1, n_{1}+n_{2}=n$. In the same way, let

$$
T_{\nu}=\left[\begin{array}{ll}
T_{\nu, 11} & T_{\nu, 12} \\
T_{\nu, 21} & T_{\nu, 22}
\end{array}\right], \text { and } F=\left[\begin{array}{ll}
F_{11} & F_{12} \\
F_{21} & F_{22}
\end{array}\right] \text {. }
$$

We also may assume $F_{22}$ to be invertible (otherwise multiply $T_{\nu}$ and $F$ from the left by an appropriately chosen permutation matrix without changing $\sigma$ ), and we conclude from (2.1) that $T_{\nu, 22}$ is invertible, at least for $|z|$ large enough, for all $\nu$. Hence we may factor

$$
T_{\nu}=\left[\begin{array}{cc}
I & T_{\nu, 12} T_{\nu, 22}^{-1} \\
0 & I
\end{array}\right]\left[\begin{array}{cc}
T_{\nu, 11}-T_{\nu, 12} T_{\nu, 22}^{-1} T_{\nu, 21} & 0 \\
T_{\nu, 21} & T_{\nu, 22}
\end{array}\right] .
$$

Since $T_{\nu, \nu-1}=T_{\nu}^{-1} T_{\nu-1}$ has a zero block in the $(1,2)$ position, then calculating the $(1,2)$ position in the product we obtain

$$
T_{\nu, 12} T_{\nu, 22}^{-1}=T_{\nu-1,12} T_{\nu-1,22}^{-1} \text { for } \quad \nu=1, \ldots, m .
$$

In light of (2.2), we see that

$$
\left[\begin{array}{cc}
I & \hat{T}_{12} \\
0 & I
\end{array}\right]=\hat{T}=\left[\begin{array}{cc}
I & T_{\nu, 12} T_{\nu, 22}^{-1} \\
0 & I
\end{array}\right]
$$


is independent of $\nu$ and defines a single-valued analytic function which is asymptotic to

$$
\left[\begin{array}{cc}
I & F_{12} F_{22}^{-1} \\
0 & I
\end{array}\right]
$$

hence $\hat{T}$ is a meromorphic transformation.

Let us therefore assume without loss of generality $T_{\nu, 12}=0$ for $\nu=0, \ldots, m$ and consequently $F_{12}=0$. Then (2.1), (2.2) imply $T_{v, j j} \cong F_{j j}$ in $S_{n}, T_{m, j j}(z)=T_{0, j j}\left(z e^{-2 \pi i}\right)$, and $T_{\nu, j j}^{-1} T_{\nu-1, j j}$ is reduced of type $\sigma_{j}^{+}$for $j=1,2$. Hence we may, by the induction hypothesis, assume that there exist actual meromorphic transformations $T_{j j}$ such that for $j=1,2$ and $\nu=0, \ldots, m$

$$
T_{\nu, j j}=T_{j j} \tilde{T}_{\nu, j}, \quad \tilde{T}_{\nu, j i} \quad \text { reduced of type } \sigma_{j}^{+} .
$$

So, splitting off a left-hand factor $T=\operatorname{diag}\left[T_{11}, T_{22}\right]$ from all the $T_{\nu}$, we may assume without loss of generality that $T_{\nu, j j}$ is reduced of type $\sigma_{j}^{+}$. In this case, we factor

and we see

$$
T_{\nu}=\left[\begin{array}{cc}
I & 0 \\
\hat{T}_{\nu, 21} & I
\end{array}\right]\left[\begin{array}{cc}
T_{\nu, 11} & 0 \\
0 & T_{\nu, 22}
\end{array}\right], \hat{T}_{\nu, 21}=T_{\nu, 21} T_{\nu, 11}^{-1},
$$

$$
T_{\nu, \nu-1}=\left[\begin{array}{cc}
T_{\nu, 11}^{-1} T_{\nu-1,11} & 0 \\
T_{\nu, 22}^{-1}\left(\hat{T}_{\nu-1,21}-\hat{T}_{\nu, 21}\right) T_{\nu-1,11} & T_{\nu, 22}^{-1} T_{\nu-1,22}
\end{array}\right] .
$$

This shows that $T_{\nu, \nu-1}$ is reduced of type $\sigma^{+}$iff $\hat{T}_{\nu-1,21}-\hat{T}_{\nu, 21}$ has non-zero elements only in positions in $\sigma$. Hence for positions not in $\sigma$, the elements of $\hat{T}_{\nu, 21}$ do not depend on $\nu$, and are therefore functions meromorphic at $z=\infty$. This proves

$$
\hat{T}_{\nu, 21}=T_{21}+\tilde{T}_{\nu, 21},
$$

where

$$
\tilde{T}_{\nu}=\left[\begin{array}{cc}
I & 0 \\
\tilde{T}_{\nu, 21} & I
\end{array}\right] \operatorname{diag}\left[T_{\nu, 11}, T_{\nu, 22}\right]
$$

is reduced of type $\sigma^{+}$.

Therefore

$$
T=\left[\begin{array}{cc}
I & 0 \\
T_{21} & I
\end{array}\right]
$$

is meromorphic at $z=\infty, T_{\nu}(z)=T(z) \tilde{T}_{\nu}(z)$, and this completes the proof of the proposition.

Remark. If the matrix $F(z)$ in Proposition 3 is formally analytic, then an inspection of the proof shows that in (2.3) we may take $T$ to be an actual analytic transformation.

Now let a fixed formal fundamental solution $H=\Psi e^{Q}$ of $[A]$ with normalised $Q$ be selected, and let $e^{2 \pi i L}$ resp. $\left(V_{\nu}\right)$ denote the corresponding formal circuit matrix resp. the corresponding normalised connection system. Then we have

Theorem 1. A meromorphic differential equation $[A]$ is meromorphically reducible of type $\sigma$ iff there exists a diagonally blocked (like $Q(z)$ ) invertible constant matrix D and a set $\tilde{\sigma}$ equivalent to $\sigma$ such that 


$$
e^{Q}, D^{-1} e^{2 \pi i L} D \text { and } D^{-1} V_{\nu} D \text { forall } \nu
$$

are reduced of type $\tilde{\sigma}^{+}$.

Remark 1. Utilising Proposition 4, Part 2 and the fact that a product of reduced matrices is again reduced of the same type, we see that it is sufficient for reducibility if $e^{Q}, D^{-1} e^{2 \pi i L} D$ and $D^{-1} V_{\nu} D$ for $\nu=1, \ldots, m$ are reduced of type $\tilde{\sigma}^{+}$.

Remark 2. Given $\tilde{\sigma}$, a matrix $C$ is reduced of type $\tilde{\sigma}^{+}$iff its elements outside $\tilde{\sigma}$ are zero off the diagonal and one on the diagonal. In other words, the position set where $C$ differs from $I$ must be contained in $\tilde{\sigma}$. There is therefore a minimal $\tilde{\sigma}$ such that the conditions of Theorem 2 for fixed $D$ are satisfied, namely the transitive closure of the position set where one of the matrices $e^{O}, D^{-1} e^{2 \pi i L} D$ and $D^{-1} V_{\nu} D$ is different from $I$. In general, the possible choices of $D$ will influence $\tilde{\sigma}$, but if $D$ is strictly diagonal this is not the case (this happens e.g. if all $s_{j}=1$ ).

Proof. (a) If $[A]$ is reducible of type $\sigma$, then $[A]$ is equivalent to a system $[\tilde{A}]$ by means of a meromorphic transformation $T$, and $\tilde{A}$ is reduced of type $\sigma$. Then $[\tilde{A}]$ has, according to Proposition 1, a formal fundamental solution $\tilde{H}=\tilde{\Psi} e^{\mathscr{O}}$, which is reduced of type $\sigma^{+}$and $\tilde{Q}$ is, perhaps, not normalised. An additional permutation similarity applied to $[\tilde{A}]$ normalises $\tilde{Q}$, and the new $\tilde{H}$ is reduced of type $\tilde{\sigma}^{+}$. Using the same notation for the new objects we conclude $\tilde{Q}=Q$ and $T \tilde{H}=H D$ for a diagonally blocked constant invertible $D$. It follows that $e^{Q}$ is reduced of type $\tilde{\sigma}^{+}$. Since $D^{-1} e^{2 \pi i L} D$ resp. $D^{-1} V_{\nu} D$ are the formal circuit matrix resp. the normalised connection matrices corresponding to $\tilde{H}$, they are reduced of type $\tilde{\sigma}^{+}$according to the Corollary of Proposition 2 and the Remark following Proposition 1 . This proves the necessity.

(b) Conversely, let $e^{Q}, D^{-1} e^{2 \pi i L} D$ and $D^{-1} V_{\nu} D$ be reduced of type $\tilde{\sigma}^{+}$for all $\nu$. Let $\tilde{H}=H D$, then $e^{2 \pi i L}=D^{-1} e^{2 \pi i L} D$ resp. $\tilde{V}_{\nu}=D^{-1} V_{\nu} D$ are the formal circuit matrix resp. the normalised connection matrices corresponding to $\tilde{H}$. We choose $\tilde{L}$ to be reduced of type $\tilde{\sigma}$ (compare the Remark following Proposition 3), and define $\tilde{F}$ by

$$
\tilde{H}=\tilde{F} z^{L} e^{Q} \text {. }
$$

Then $\tilde{F}$ is a formal meromorphic series.

If $\tilde{X}_{\nu}$ denote the basic solutions corresponding to $\tilde{H}$, we define

$$
\tilde{T}_{\nu}=\tilde{X}_{\nu} e^{-Q} z^{-i}
$$

and we have (in view of $\tilde{X}_{m}(z)=\tilde{X}_{0}\left(z e^{-2 \pi i}\right) e^{2 \pi i L}, \tilde{H}(z)=\tilde{H}\left(z e^{-2 \pi i}\right) e^{2 \pi i L}$ )

$$
\begin{aligned}
& \tilde{T}_{\nu}(z) \cong \tilde{F}(z) \text { in } S_{\nu} \quad \text { for } \quad \nu=0, \ldots, m \text {, } \\
& \begin{aligned}
\tilde{T}_{m}(z) & =\tilde{T}_{0}\left(z e^{-2 \pi i}\right) \quad \text { in } \quad S_{m}, \\
\tilde{T}_{\nu, \nu-1}(z) & =\tilde{T}_{\nu}^{-1}(z) \tilde{T}_{\nu-1}(z)=z^{L} e^{Q} \tilde{V}_{\nu} e^{-Q} z^{-\bar{L}}, \quad(\nu=1, \ldots, m),
\end{aligned}
\end{aligned}
$$

and $\tilde{T}_{\nu, \nu-1}$ is reduced of type $\tilde{\sigma}^{+}$. If we apply Proposition 3 , then $\tilde{T}_{\nu}(z)=T(z) \hat{T}_{\nu}(z)$, where $T(z)$ is a meromorphic transformation, and $\hat{T}_{\nu}$ are reduced of type $\tilde{\sigma}^{+}$.

But $\hat{X}_{\nu}=T^{-1} \bar{X}_{\nu}=\hat{T}_{\nu}(z) z^{L} e^{O}$ is also reduced of type $\tilde{\sigma}^{+}$, therefore its logarithmic derivative $\hat{A}$ is reduced of type $\tilde{\sigma}$. Thus $T$ takes $[A]$ into $[\hat{A}]$ and an additional permutation takes $[\hat{A}]$ into a reduced differential equation of type $\sigma$. This completes the proof. 
Remark. The given formal solution $H$ of $[A]$ may be factored as

$$
H=F_{a} P(z) z^{K} z^{L} e^{Q} ;
$$

here $P(z) z^{K}$ are as in Lemma 2 of (1) and uniquely determined once $L$ is selected, and we assume that the eigenvalues of $L$ belong to a fixed system of representatives mod 1 , where the zero equivalence class is represented by zero. For example, if $H=H_{a}$, then $P(z) z^{K}$ are the matrices which were selected to form a complete system of formal analytic invariants. Then, using the proof of Theorem 1 and the Remarks following Proposition 1 resp. Proposition 3, we see:

A meromorphic differential equation $[A]$ is analytically reducible of type $\sigma$ iff there exists $a$ diagonally blocked invertible constant matrix $D$ and a set $\tilde{\sigma}$ equivalent to $\sigma$ such that

$$
e^{O}, D^{-1} e^{2 \pi i L} D \text { and } D^{-1} V_{\nu} D \text { forall } \nu
$$

are reduced of type $\tilde{\sigma}^{+}$, and additionally there exists a factorisation of

$$
P(z) z^{K} D=F_{a}(z) \tilde{P} z^{\tilde{K}}
$$

where $F_{a}(z)$ is an analytic transformation, $\tilde{P}(z)$ a matrix polynomial such that $\operatorname{diag} \tilde{P}(z)=I$, $\tilde{P}(0)=I, \tilde{K}$ is a diagonal matrix of integers, and $\tilde{P}(z) z^{K}$ is reduced of type $\tilde{\sigma}^{+}$.

Note that $(2.4)$ is automatically satisfied if $(i, j)$ or $(j, i)$ belongs to $\sigma$ for every pair $(i, j)$, so that in this case meromorphic and analytic reducibility are equivalent.

We generalise the notion of reducibility in a natural way as follows:

A meromorphic differential equation $[A]$ is said to be $v$-meromorphically reducible if there exists an actual $v$-meromorphic transformation which takes $[A]$ into a system $[\tilde{A}]$, where $\tilde{A}$ in general is a $v$-meromorphic matrix and reduced of type $\sigma$ for some non-trivial transitive position set $\sigma$.

Corollary to Theorem 1. A meromorphic differential equation $[A]$ is $v$ meromorphically reducible of type $\sigma$ iff there exists a diagonally blocked invertible constant matrix $D$ and a set $\tilde{\sigma}$ equivalent to $\sigma$ such that

$$
e^{Q}, D^{-1} e^{2 v \pi i L} D \text { and } D^{-1} V_{\nu} D \text { forall } \nu
$$

are reduced of type $\tilde{\sigma}^{+}$.

A proof of this Corollary in a special case can be found in $(6, p .149)$, and in just the same way the general case can be derived, using the general form of Theorem 1 . We therefore omit the details of the proof.

Remark 1. Again it is sufficient for $v$-meromorphic reducibility to require that

$$
e^{Q}, D^{-1} e^{2 v \pi i L} D \text { and } D^{-1} V_{\nu} D \text { for } \nu=1, \ldots, m v
$$

are reduced of type $\tilde{\sigma}^{+}$.

Remark 2. In many examples, the conditions for $v$-meromorphic reducibility imply meromorphic reducibility of a possibility different type. There are, however, cases of equations which are $v$-meromorphically reducible, but not meromorphically reducible of any type. For an example, note that meromorphic reducibility implies that $Q$ can be split 
into at least two parts which are closed under analytic continuation. Hence if we take $n=4$ and $Q$ consisting of a single superblock with $p=4, s=1$, the corresponding equation cannot be meromorphically reducible, independent of what the proper invariants are. However it may be $v$-meromorphically reducible for $v=2,4$ (take, for example, the connection matrices to be $I$ ).

\section{Convergent formal vector solutions}

Let $h(z)$ be a column vector of formal logarithmic-exponential expressions which satisfies a given equation [A]. We call $h(z)$ a convergent vector solution, if all the formal series in the reduced forms of the components of $h(z)$ are convergent.

Let now $[A]$ be given. The set of all convergent vector solutions of $[A]$ is a vector space of dimension, say $k, 0 \leqq k \leqq n$, which is closed under analytic continuation and which has, in case $k \geqq 1$, a basis $h_{1}, \ldots, h_{k}$.

If $h$ is any formal or convergent solution vector of $[A]$ then it has the form

$$
h(z)=H c
$$

where $H(z)=\Psi(z) e^{O(z)}$ is a formal fundamental solution and $c$ a constant vector. Therefore we have

$$
h(z)=\psi_{1}(z) e^{q_{1}(z)}+\ldots+\psi_{l}(z) e^{q_{l}(z)},
$$

and $\psi_{j}$ is a linear combination of columns of $\Psi$ corresponding to $q_{j}$. Hence every $\psi_{j} e^{q_{j}}$ is again a solution of [A] and converges if $h(z)$ converges. Therefore we may without loss of generality assume that the selected basis consists of vectors $h_{j}=\psi_{j} e^{q_{i}}(1 \leqq j \leqq k)$. Selecting further linearly independent divergent $h(z)$, we can construct a formal fundamental solution

$$
\tilde{H}=\tilde{\Psi} e^{\tilde{Q}},
$$

where the first $k$ columns of $\tilde{H}$ equal $h_{1}, \ldots, h_{k}$, and $\tilde{Q}$ is not necessarily normalised.

Since the space of all convergent vector solutions is closed under analytic continuation, the analytic continuation of $h_{j}(1 \leqq j \leqq k)$ depends linearly on $h_{1}, \ldots, h_{k}$, hence the formal monodromy factor $e^{2 \pi i L^{-}}$of $H$ is reduced of type $\sigma_{k}^{+}$, where

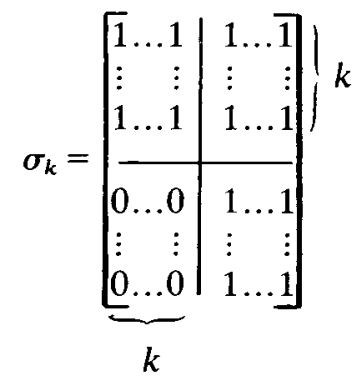

Therefore let without loss of generality $\tilde{L}$ be selected to be reduced of type $\sigma_{k}$, then (since $z^{\bar{L}}$ then is reduced of type $\sigma_{k}^{+}$) we have

$$
\tilde{H}=\tilde{F} z^{\tilde{L}} e^{\tilde{Q}}
$$


where $\tilde{F}$ is formally meromorphic such that the first $k$ columns of $\tilde{F}$ converge and $L$ is reduced of type $\sigma_{k}$.

Every fundamental formal solution $\tilde{H}$ of the form (3.1) for some $k(1 \leqq k \leqq n)$ may be called partially convergent, and $k$ may be called the rank of convergence. It is clear that a partially convergent $\tilde{H}$ gives rise to $k$ linearly independent convergent vector solutions, namely its first $k$ columns, and if we find a partially convergent $\tilde{H}$ where the corresponding $k$ is maximal, then every convergent vector solution is a linear combination of the first $k$ columns of $\tilde{H}$. The problem of finding all convergent vector solutions is therefore reduced to finding a partially convergent $\tilde{H}$ with maximal $k$, and the following theorem will characterise all partially convergent $\tilde{H}$ in terms of invariants.

To do this, let $H=\Psi e^{Q}$ with normalised $Q$ be a fixed formal fundamental solution of $[A]$, with corresponding formal circuit matrix $e^{2 \pi i L}$, normalised connection system $\left(V_{v}\right)$, and normal solutions $X_{\nu}$. Let finally $\tilde{\sigma}_{k}$ be the transitive set with the incidence matrix

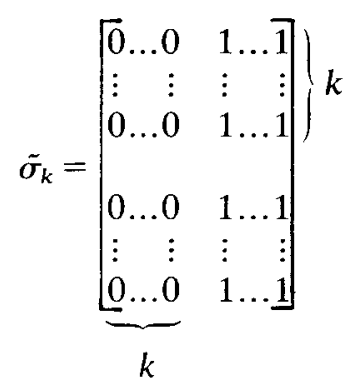

Then we have

Theorem 2. The differential equation $[A]$ has a partially convergent formal fundamental solution $\hat{H}$ of rank $k$ iff it is reducible of type $\sigma_{k}$ to $[\hat{A}]$,

$$
\hat{A}=\left[\begin{array}{cc}
\hat{A}_{11} & \hat{A}_{12} \\
0 & \hat{A}_{22}
\end{array}\right],
$$

where $\left[\hat{A}_{11}\right]$ is a $k \times k$ matrix having a convergent formal solution. Furthermore, a formal fundamental solution $\tilde{H}=\tilde{\Psi} e^{\tilde{O}}$ is partially convergent of rank $k$ iff it is of the form

$$
\tilde{H}=H C \text {, }
$$

where $C=D R$ with $D$ a constant, diagonally blocked (like $Q$ ) and invertible matrix, $R$ a suitable permutation matrix, such that

$$
C^{-1} e^{2 \pi i L} C \text { is reduced of type } \sigma_{k}^{+} \text {and } C^{-1} V_{\nu} C \text { is reduced of type } \tilde{\sigma}_{k}^{+}(3.4)
$$

for all $\nu$. Finally, for these $C$ the first $k$ columns of $X_{\nu} C$ (the normal solutions corresponding to $\tilde{H})$ are equal to the same columns of $\tilde{H}=H C$ and a transformation reducing $[A]$ can be explicitly constructed using these columns.

Proof. Let $[A]$ have a partially convergent solution $\tilde{H}=\tilde{F} z^{L} e^{\tilde{Q}}$ of $\operatorname{rank} k$. Then

$$
\tilde{F}=\left[\begin{array}{ll}
\tilde{F}_{11} & \tilde{F}_{12} \\
\tilde{F}_{21} & \tilde{F}_{22}
\end{array}\right],
$$


where $\tilde{F}_{11}$ is a $k \times k$ matrix and, without loss of generality, we assume $\tilde{F}_{11}$ to be invertible (otherwise apply a permutation to the rows of $\tilde{H}$ which corresponds to a permutation similarity of $[A])$. Then we factor

$$
\tilde{F}=T \hat{F}=\left[\begin{array}{cc}
\tilde{F}_{11} & 0 \\
\tilde{F}_{21} & I
\end{array}\right]\left[\begin{array}{cc}
I & \tilde{F}_{11}^{-1} \tilde{F}_{12} \\
0 & \tilde{F}_{22}-\tilde{F}_{21} \tilde{F}_{11}^{-1} \tilde{F}_{12}
\end{array}\right],
$$

and $T$ is an actual meromorphic transformation which takes $(A, H)$ into $(\hat{A}, \hat{H})$ where

$$
\hat{H}=\hat{F} z^{L} e^{\tilde{Q}}
$$

is reduced of type $\sigma_{k}^{+}$and has a convergent block in the first diagonal position. Therefore $[\hat{A}]$ is reduced of type $\sigma_{k}$, hence of the form (3.2) with [ $\left.\hat{A}_{11}\right]$ having the convergent formal solution $z^{\bar{L}_{11}} e^{\bar{Q}_{1}}$. Note that the transformation $T$ is explicitly determined by the first $k$ columns of $\tilde{H}$. Furthermore, there is a unique $C=D R$ with $D$ and $R$ as described in the Theorem satisfying (3.3), and the pairs $(A, H C=\tilde{H})$ and $(\hat{A}, \hat{H})$ have the same invariants, namely $C^{-1} e^{2 \pi i L} C$ and $C^{-1} V_{\nu} C$. Using the Corollary of Proposition 2 we therefore obtain (3.4) for this $C$.

Now assume $[A]$ to be reducible of type $\sigma_{k}$ to an [Â], satisfying (3.2). Then according to Proposition 1 there exists a formal fundamental solution

$$
\hat{H}=\left[\begin{array}{cc}
\hat{H}_{11} & \hat{H}_{12} \\
0 & \hat{H}_{22}
\end{array}\right]=\hat{F}_{m} z^{\hat{L}} e^{\hat{Q}},
$$

where $\hat{H}_{11}$ is convergent and $\hat{L}$ is reduced of type $\sigma_{k}$. Let $T$ be any actual meromorphic transformation taking $[A]$ into $[\hat{A}]$. Then

$$
\tilde{H}=T \hat{H}
$$

is a formal fundamental solution of $[A]$ which is partially convergent of rank $k$.

Now let (3.4) be satisfied for some $C$ as described in the Theorem. Then $\tilde{X}_{\nu}=X_{\nu} C$, $e^{2 \pi i \bar{L}}=C^{-1} e^{2 \pi i L} C$, and $\tilde{V}_{\nu}=C^{-1} V_{\nu} C$ are the normal solutions resp. the formal circuit matrix resp. the normalised connection matrices corresponding to $\tilde{H}=H C=\tilde{\Psi} e^{\tilde{O}}$. From $\tilde{X}_{\nu-1}=\tilde{X}_{\nu} \tilde{V}_{\nu}$ and the fact that $\tilde{V}_{\nu}$ is reduced of type $\tilde{\sigma}_{k}^{+}$we conclude that the first $k$ columns of $\tilde{X}_{\nu}$ do not depend on $\nu$. From $\tilde{X}_{m}(z)=\tilde{X}_{0}\left(z e^{-2 \pi i}\right) e^{2 \pi i \tilde{L}}$ and the fact that $e^{2 \pi i \tilde{L}}$ is reduced of type $\sigma_{k}^{+}$(so that we can choose $\tilde{L}$ to be reduced of type $\sigma_{k}$ ) we see that the first $k$ columns of $\tilde{X}_{\nu} e^{-\tilde{Q}_{z}} z^{-\bar{L}}$ are single valued functions in the $z$-plane having as an asymptotic in a full neighbourhood of $\infty$ the first $k$ columns of $\tilde{F}=\tilde{H} e^{-\bar{Q}} z^{-\bar{L}}$. Therefore these columns of $\tilde{F}$ converge, hence $\tilde{H}$ is partially convergent of rank $k$, and the first $k$ columns of $\tilde{X}_{\nu}$ are equal to the corresponding columns of $\tilde{H}$. This completes the proof.

Remark. In (6) we considered a related, but slightly differently phrased question, namely whether $[A]$ has a formal fundamental solution $H=F_{w} z^{j} e^{Q}$ (where $z^{j} e^{Q}$ is the formal root-meromorphic invariant and $F_{w}$ a formal root-meromorphic series) exists with at least one column of $F_{w}$ convergent. This then implied the existence of several linearly independent convergent formal vector solutions, but did not necessarily give all the convergent formal vector solutions. 


\section{Calculation of invariants for reduced systems}

In (2) we remarked that the Closing Theorem guarantees the computability of the invariants. However, an actual computation requires the knowledge of actual solutions $\hat{X}_{\nu} \cong H$ in $S_{\nu}$ (for some formal fundamental solution $H$ ) and setting $Y_{\nu}=\hat{X}_{\nu} C_{\nu}$ and using the Closing Theorem leads then to a non-linear system of equations for the $C_{\nu}$. The situation becomes considerably easier in the case of a differential equation $[A]$ which is reduced of type $\sigma$, where we assume that $\sigma$ is normalised and the normal solutions of the diagonal blocks are known. We will see that in this case some $\hat{X}_{\nu}$ can be constructed by quadrature and the system of equations for $C_{\nu}$ becomes linear. Throughout this section, let $\sigma$ have the following incidence matrix

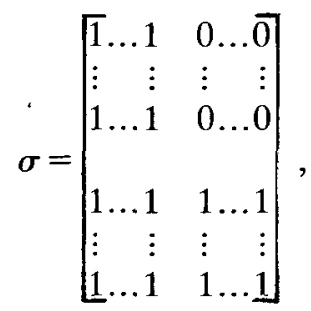

where the diagonal blocks of $\sigma$ are of sizes $n_{j} x n_{j}$, say, with $1 \leqq n_{j}, n_{1}+n_{2}=n$. A generalisation to arbitrary (normalised) $\sigma$ can be obtained by an application of the following discussion to each diagonal block.

Let $A(z)$ be reduced of type $\sigma$, then

$$
A(z)=\left[\begin{array}{cc}
A_{11}(z) & 0 \\
A_{21}(z) & A_{22}(z)
\end{array}\right]
$$

and let

$$
H(z)=\left[\begin{array}{cc}
H_{11}(z) & 0 \\
H_{21}(z) & H_{22}(z)
\end{array}\right]=\left[\begin{array}{cc}
\Psi_{11}(z) & 0 \\
\Psi_{21}(z) & \Psi_{22}(z)
\end{array}\right] \exp \left(\operatorname{diag}\left[Q_{1}(z), Q_{2}(z)\right]\right)
$$

be a fixed formal fundamental solution of $[A]$ which is reduced of type $\sigma$, too. Then for $j=1,2, H_{j j}(z)$ is a formal fundamental solution of $\left[A_{j j}\right]$, and we assume that the corresponding normal solutions $X_{\nu, j j}$ are known. By $V_{\nu, j j}$ we denote the corresponding connection matrices (see the Corollary to Proposition 2).

From Section 1 we know that the normal solutions $X_{\nu}$ of $[A]$ corresponding to $H$ have the form

and by "variations of constants"

$$
X_{\nu}=\left[\begin{array}{cc}
X_{\nu, 11} & 0 \\
X_{\nu, 21} & X_{\nu, 22}
\end{array}\right]
$$

$$
X_{\nu, 21}(z)=X_{\nu, 22}(z) \int X_{\nu, 22}^{-1}(z) A_{21}(z) X_{\nu, 11}(z) d z
$$

if we choose the antiderivative of $X_{\nu, 22}^{-1} A_{21} X_{\nu, 11}$ appropriately. Choosing $z_{0}$ on the Riemann surface of $\log z$ arbitrarily (but $\left|z_{0}\right|$ large enough), then all the possible antiderivatives are of the form

$$
Y_{\nu, 21}(z)=\int_{z^{0}}^{z} X_{\nu, 22}^{-1}(\zeta) A_{21}(\zeta) X_{\nu, 11}(\zeta) d \zeta+C_{\nu, 21}
$$


with a constant matrix $C_{\nu, 21}$. We see that $X_{\nu, 22} Y_{\nu, 21} \cong \Psi_{21} e^{Q_{1}}$ in $S_{\nu}$ is equivalent to

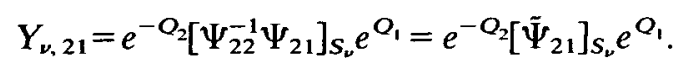

Let the elements of matrices with subscripts 2,1 be denoted by corresponding lower case letters, indexed by subscripts $j, k$, where $n_{1}+1 \leqq j \leqq n, 1 \leqq k \leqq n_{1}$. Then we see that

$$
y_{\nu, j k}(z) \rightarrow 0 \quad \text { as } \quad z \rightarrow \infty, \quad \arg z=\tau,
$$

if $\tau \neq \tau_{\nu}$ is chosen such that

$$
\tau_{\nu-1}<\tau<\tau_{\nu+1}, e^{q_{k}(z)-q_{j}(z)} \rightarrow 0 \quad \text { as } \quad z \rightarrow \infty, \arg z=\tau .
$$

This implies (if $f_{\nu, j k} e^{q_{k}-q_{j}}$ denotes the $(j, k)$ element of $X_{\nu, 22}^{-1} A_{21} X_{\nu, 11}$ )

$$
c_{\nu, j k}=\int_{\Gamma_{\nu, k}} f_{\nu, j k}(\zeta) e^{q_{k}(\zeta)-q_{j}(\zeta)} d \zeta
$$

where $\Gamma_{\nu, k}$ denotes some path from $\infty$ to $z_{0}$ following the ray $\arg z=\tau$ for $|z|$ large.

If there exists no $\tau$ satisfying (4.4), then either $e^{q_{k}(z)-q_{j}(z)} \rightarrow \infty$ in $S_{\nu}$ or $q_{k}=q_{j}$. In the first case (4.2) holds whatever the value of $c_{\nu, j k}$ may be. In the second case we have $y_{\nu, j k}=\left[\tilde{\psi}_{j k}\right]_{S_{\nu}}$, which determines $c_{\nu, j k}$ uniquely (because $c=[0]_{S_{\nu}}$ implies $c=0$ for a constant $c$ ).

From

we see

$$
V_{\nu}=X_{\nu}^{-1} X_{\nu-1}=\left[\begin{array}{cc}
V_{\nu, 11} & 0 \\
V_{\nu, 21} & V_{\nu, 22}
\end{array}\right]
$$

$$
V_{\nu, 21}=V_{\nu, 22} Y_{\nu-1,21}-Y_{\nu, 21} V_{\nu, 11}
$$

hence from

$$
\begin{aligned}
V_{\nu, 22} \int_{2^{0}}^{z} X_{\nu-1,22}^{-1}(\zeta) A_{21}(\zeta) X_{\nu-1,11}(\zeta) d \zeta & =\int_{z_{0}}^{z} X_{\nu, 22}^{-1}(\zeta) A_{21}(\zeta) X_{\nu-1,11}(\zeta) d \zeta \\
& =\int_{z_{0}}^{z} X_{\nu, 22}^{-1}(\zeta) A_{21}(\zeta) X_{\nu, 11}(\zeta) d \zeta V_{\nu, 11}
\end{aligned}
$$

we obtain

$$
V_{\nu, 21}=V_{\nu, 22} C_{\nu-1,21}-C_{\nu, 21} V_{\nu, 11}
$$

From (4.7) we see that $V_{\nu} \in \mathcal{U}\left(\rho_{\nu}\right)$ means that certain linear equations in the elements of $C_{\nu, 21}$ and $C_{\nu-1,21}$ must be satisfied. These have a unique solution, if we consider the Closing Condition

$$
X_{m}(z)=X_{0}\left(z e^{-2 \pi i}\right) e^{2 \pi i L}
$$

which relates $C_{m, 21}$ to $C_{0,21}$.

In a simple case we can solve this system explicitly in terms of contour integrals over the elements of $X_{\nu, 22}^{-1} A_{21} X_{\nu, 11}$ for $\nu=0, \ldots, m-1$ :

Let the leading coefficient of $A(z)$ have all distinct eigenvalues. Then (see (3)) we know that

$$
X_{\nu} \cong H \text { in } \mathscr{S}_{\nu}
$$


where $\mathscr{S}_{\nu}$ is a "large" sector extending from $\tau_{\nu+1}$ in the negative direction, until every pair $(j, k)$ changes dominance in $\mathscr{P}_{\nu}$ exactly once. Then, according to $(4.5)$, the matrix $C_{\nu, 21}$ is explicitly constructable in terms of integrals along certain paths from $\infty$ to $z_{0}$, and so is $Y_{\nu, 21}(z)$ (take $z=z_{0}$ ). If we additionally assume that $V_{\nu, 21} \neq 0$ only if $V_{\nu, j j}=I_{j}$ for $j=1,2$ (which is, for example, true if all the $\rho_{\nu}$ are only singletons), then for those $\nu$ where $V_{\nu, 21} \neq 0$, we have $X_{\nu-1, j j}=X_{\nu, j j}$ for $j=1,2$, hence (4.6) implies

$$
V_{\nu, 21}=\int_{\Gamma_{\nu-1}}^{z} X_{\nu, 22}^{-1}(\zeta) A_{21}(\zeta) X_{\nu, 11}(\zeta) d \zeta-\int_{\Gamma_{\nu}}^{z} X_{\nu, 22}^{-1}(\zeta) A_{21}(\zeta) X_{\nu, 11}(\zeta) d \zeta
$$

where $\Gamma_{\mu}$ generically denotes paths from $\infty$ to $z$ which may be different for the elements of the integrand and are chosen according to (4.5), and the elements in $V_{\nu, 21}$ which are non-zero are given as a contour integral over the corresponding element of $X_{\nu, 22}^{-1} A_{21} X_{\nu, 11}$ along a contour indicated in Figure 1.

We summarise these results as follows.

Proposition 4. Suppose $[A]$ is reduced of type $\sigma$, and that a reduced formal fundamental solution $H$ and the diagonal blocks of the corresponding normal solutions and connection matrices are known. Then the $(2,1)$ blocks of $X_{\nu}$ and $V_{\nu}$ can be explicitly calculated from $A_{21}$ using constant matrices $C_{\nu, 21}$ as follows:

$$
\begin{aligned}
X_{\nu, 21}(z) & =X_{\nu, 22}(z)\left[\int_{z^{0}}^{z} X_{\nu, 22}^{-1}(\zeta) A_{21}(\zeta) X_{\nu, 11}(\zeta) d \zeta+C_{\nu, 21}\right] \\
V_{\nu, 21} & =V_{\nu, 22} C_{\nu-1,21}-C_{\nu, 21} V_{\nu, 11} .
\end{aligned}
$$

Some of the elements of $C_{\nu, 21}$ are determined by the required asymptotic for $X_{\nu, 21}$ (using integrals extending to $\infty$ ) and the others by the support conditions for $V_{\nu, 21}$ (which represent linear equations). In the special case of different eigenvalues and "simple" Stokes' rays, e.g., the invariants $V_{\nu, 21}$ are given by explicit loop integrals.

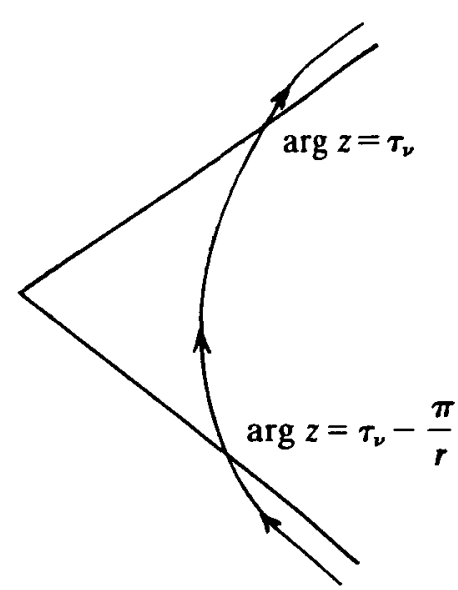

Fig. 1 


\section{Construction of reduced differential equations}

We now assume that matrices $Q, L$ and $V_{\nu}$ (for all $\nu$ ) are given which correspond to a reducible differential equation of type $\sigma$ and that the diagonal blocks $X_{\nu, j j}$ of the normalised solutions $X_{\nu}$ are known and are of the form

$$
X_{\nu, j j}=E_{\nu, j j} z^{M_{\nu, j}}
$$

with a constant matrix $M_{\nu, j}$ and a matrix $E_{\nu, j j}$ of entire functions with non-zero determinant for all $z$. If $X_{\nu, j j}$ does not directly satisfy (5.1), then we can modify it by a meromorphic transformation; see (6). Such transformations can also be used to change the eigenvalues of $M_{\nu, j} \bmod 1$, and we will use this freedom later to ensure the convergence of certain integrals.

Our objective is to obtain an explicit integral representation for matrices $X_{\nu, 21}$ which together with $X_{\nu, j j}$ form normal solutions $X_{\nu}$ of a differential equation [A] which is reduced of type $\sigma$ and has the prescribed matrices as invariants.

Let

$$
\begin{aligned}
T_{\nu, \nu-1}=\operatorname{diag}\left[X_{\nu, 11} e^{-Q_{1}} z^{-L_{11}}, X_{\nu, 22} e^{-Q_{2}} z^{-L_{22}}\right] z^{L} e^{Q} V_{\nu} e^{-Q_{2}-L} & \\
& \operatorname{diag}\left[z^{L_{11}} e^{Q_{1}} X_{\nu-1,11}^{-1}, z^{L_{22}} e^{Q_{2}} X_{\nu-1,22}^{-1}\right] .
\end{aligned}
$$

Then $T_{\nu, \nu-1}(z)$ has $I_{n_{j}}$ as matrices on the diagonal and $T_{\nu, \nu-1} \cong I$ in $S_{\nu}^{\prime}$. The $(2,1)$ block of $T_{\nu, \nu-1}$ can be written as follows:

$$
T_{\nu, \nu-1,21}^{\cdot}=X_{\nu, 22}(z)\left[V_{\nu, 21}+\mathscr{L}_{21}(z) V_{\nu, 11}-V_{\nu, 22} \mathscr{L}_{21}(z)\right] X_{\nu-1,11}^{-1}(z) ;
$$

where $\mathscr{L}_{21}(z)$ denotes the $(2,1)$ block of $\operatorname{diag}\left[e^{-Q_{1}} z^{-L_{11}}, e^{-O_{2}} z^{-L_{22}}\right] z^{L} e^{Q}$. By adding a suitably large integer to all the eigenvalues of $M_{\nu, 2}$ (for all $v$ ), we can arrange that expression (5.2) tends to zero as $z$ tends to zero along a fixed, but arbitrary ray. Therefore, if we define $\gamma_{\nu}=\left\{\zeta|0 \leqq| \zeta \mid<\infty, \arg \zeta=1 / 2\left(\tau_{\nu-1}+\tau_{\nu}\right)\right\}$ directed from 0 to $\infty$, we have that

$$
T_{\nu}(z)=I-\frac{1}{2 \pi i} \sum_{\mu=1}^{m} \int_{\gamma_{\mu}} \frac{1}{\zeta-z}\left(T_{\mu, \mu-1}(\zeta)-I\right) d \zeta, \text { for }\left|\arg z-\tau_{\nu}\right| \text { small, }
$$

defines matrix functions which are analytic near the ray $\arg z=\tau_{n}$ and can be analytically extended on the whole Riemann surface of $\log z$ (by deforming the path of integration appropriately).

Obviously

$$
T_{\nu}(z)=\left[\begin{array}{cc}
I & 0 \\
T_{\nu, 21}(z) & I
\end{array}\right]
$$

and clearly by definition (using that $\frac{1}{\zeta-z}$ is single valued) $T_{\nu+m}(z)=T_{\nu}\left(z e^{-2 \pi i}\right)$.

Furthermore, we see from

that

$$
\frac{1}{\zeta-z}=-\sum_{j=1}^{k} \frac{\zeta^{j-1}}{z^{j}}+\frac{1}{z^{k}} \frac{\zeta^{k}}{\zeta-z}(z \neq 0, \zeta ; k=1,2, \ldots)
$$

$$
T_{\nu}(z)=I+\sum_{i=1}^{k} \tilde{F}_{j} z^{-i}+z^{-k} R_{\nu, k}(z),
$$


where

$$
\begin{gathered}
\tilde{F}_{j}=\frac{1}{2 \pi i} \sum_{\mu=1}^{m} \int_{\gamma_{\mu}} \zeta^{j-1}\left(T_{\mu, \mu-1}(\zeta)-I\right) d \zeta \\
R_{\nu, k}(z)=\frac{-1}{2 \pi i} \sum_{\mu=1}^{m} \int_{\gamma_{\mu}} \frac{\zeta^{k}}{\zeta-z}\left(T_{\mu, \mu-1}(\zeta)-I\right) d \zeta
\end{gathered}
$$

From $T_{\mu \mu-1}(\zeta) \cong I$ in $S_{\mu}^{\prime}$ we conclude (by possibly deforming the path of integration in case $z \in \gamma_{\mu}$ ) that $R_{\nu, k}(z) \rightarrow 0$ as $z \rightarrow \infty$, uniformly in every proper subsector of $S_{\nu}$, hence $T_{\nu}$ admits an asymptotic power series expansion in $S_{n}$ and the coefficients are given by (5.6).

In order to calculate $T_{\nu}^{-1} T_{\nu-1}$, we first remark that

$$
T_{\nu+m, \nu+m-1}(z)=T_{\nu, \nu-1}\left(z e^{-2 \pi i}\right),
$$

which implies

$$
\int_{\gamma_{\mu+m}} \frac{1}{\zeta-z}\left(T_{\mu+m, \mu+m-1}(\zeta)-I\right) d \zeta=\int_{\gamma_{\mu}} \frac{1}{\zeta-z}\left(T_{\mu, \mu-1}(\zeta)-I\right) d \zeta .
$$

Therefore we have for any integer $k$ and for small $\left|\arg z-\tau_{\nu}\right|$

$$
T_{\nu}(z)=I-\frac{1}{2 \pi i} \sum_{\mu=k}^{m-1+k} \int_{\gamma_{\mu}} \frac{1}{\zeta-z}\left(T_{\mu, \mu-1}(\zeta)-I\right) d \zeta
$$

We now take for a fixed $\nu$ some $k$ such that $k \leqq \nu \leqq m-1+k$. Then $T_{\nu}(z)$ is directly defined by (5.10) for some $z_{0}$, arg $z_{0}=\tau_{\nu}$, whereas $T_{\nu-1}(z)$ can be analytically continued to $z_{0}$ by a deformation of $\gamma_{\nu}$ into $\tilde{\gamma}_{\nu}$ (see Figure 2).

This shows

$$
\begin{aligned}
T_{\nu-1}\left(z_{0}\right)-T_{\nu}\left(z_{0}\right) & =\frac{1}{2 \pi i}\left(\int_{\gamma_{\nu}}-\int_{\bar{\gamma}_{\nu}}\right) \frac{1}{\zeta-z_{0}}\left(T_{\nu, \nu-1}(\zeta)-I\right) d \zeta \\
& =T_{\nu, \nu-1}\left(z_{0}\right)-I,
\end{aligned}
$$

and using this together with (5.4) we conclude that for all $\nu$ and $z \neq 0$

$$
T_{\nu}^{-1}(z) T_{\nu-1}(z)=I+T_{\nu-1}(z)-T_{\nu}(z)=T_{\nu, \nu-1}(z) .
$$

We now define

$$
\begin{gathered}
X_{\nu}=T_{\nu} \operatorname{diag}\left[X_{\nu, 11} e^{-Q_{1}} z^{-L_{11}}, X_{\nu, 22} e^{\left.-Q_{2} z^{-L_{22}}\right] z^{L} e^{Q}}\right. \\
=\left[\begin{array}{cc}
X_{\nu, 11} & 0 \\
X_{\nu, 21} & X_{\nu, 22}
\end{array}\right],
\end{gathered}
$$

and we see that the definition of $T_{\nu, \nu-1}$ and (5.11) imply $X_{\nu}^{-1} X_{\nu-1}=V_{\nu}$. Letting $H_{j j}=F_{j j} z^{L_{i j}} e^{Q_{j}}$ denote the asymptotic expansion of $X_{\nu, j j}$ in $S_{\nu}$, we see using (5.5), (5.6) and defining

$$
H(z)=\left(I+\sum_{1}^{\infty} \tilde{F}_{j} z^{-j}\right) \operatorname{diag}\left[F_{11}, F_{22}\right] z^{L} e^{Q}
$$

that

$$
X_{\nu} \cong H \text { in } S_{\nu},
$$




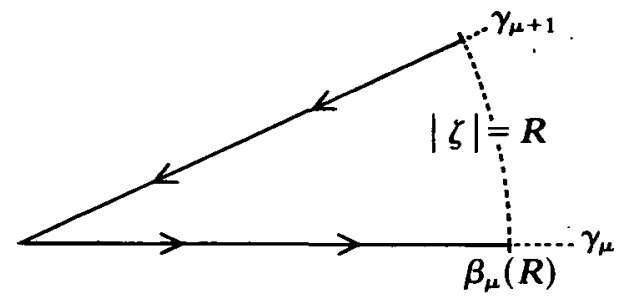

Fig. 2

hence $[A]=\left[X_{\nu}^{\prime} X_{\nu}^{-1}\right]$ is a meromorphic differential equation with $H$ as a formal solution and $X_{\nu}$ resp. $V_{\nu}$ as the corresponding normal solutions resp. connection matrices. From (5.8) we see that

$$
\begin{aligned}
A=X_{\nu}^{\prime} X_{\nu}^{-1}= & T_{\nu}^{\prime} T_{\nu}^{-1}+T_{\nu} \operatorname{diag}\left[A_{11}, A_{22}\right] T_{\nu}^{-1} \\
& +T_{\nu} \operatorname{diag}\left[X_{\nu, 11}, X_{\nu, 22}\right]\left[\begin{array}{cc}
0 & 0 \\
\mathscr{L}_{21}^{\prime}(z) & 0
\end{array}\right] \operatorname{diag}\left[X_{\nu, 11}^{-1}, X_{\nu, 22}^{-1}\right] T_{\nu}^{-1}
\end{aligned}
$$

which implies $\quad A_{21}=T_{\nu, 21}^{\prime}+T_{\nu, 21} A_{11}-A_{22} T_{\nu, 21}+X_{\nu, 22} \mathscr{L}_{21}^{\prime} X_{\nu, 11}^{-1}$.

Using (5.2), we see by differentiation of (5.3) and a partial integration (note that $T_{\mu, \mu-1,21}(\zeta) \rightarrow 0$ as $\zeta \rightarrow 0$ along arbitrary rays):

$$
\begin{aligned}
T_{\nu, 21}^{\prime}(z)= & -\frac{1}{2 \pi i} \sum_{\mu=1}^{m} \int_{\gamma_{\mu}} \frac{1}{\zeta-z}\left\{A_{22}(\zeta) T_{\mu, \mu-1,21}(\zeta)-T_{\mu, \mu-1,21}(\zeta) A_{11}(\zeta)\right\} d \zeta \\
& -\frac{1}{2 \pi i} \sum_{\mu=1}^{m} \int_{\gamma_{\mu}} \frac{1}{\zeta-z} X_{\mu, 22}(\zeta)\left\{\mathscr{L}_{21}^{\prime}(\zeta) V_{\mu, 11}-V_{\mu, 22} \mathscr{L}_{21}^{\prime}(\zeta)\right\} X_{\mu-1,11}^{-1}(\zeta) d \zeta
\end{aligned}
$$

and from (5.4) we conclude

$$
\begin{aligned}
A_{21}(z)= & -\frac{1}{2 \pi i} \sum_{\mu=1}^{m} \int_{\gamma_{\mu}} \frac{1}{\zeta-z}\left(A_{22}(\zeta)-A_{22}(z)\right) T_{\mu, \mu-1,21}(\zeta) d \zeta \\
& +\frac{1}{2 \pi i} \sum_{\mu=1}^{m} \int_{\gamma_{\mu}} \frac{1}{\zeta-z} T_{\mu, \mu-1,21}(\zeta)\left(A_{11}(\zeta)-A_{11}(z)\right) d \zeta \\
& -\frac{1}{2 \pi i} \sum_{\mu=1}^{m} \int_{\gamma_{\mu}} \frac{1}{\zeta-z} X_{\mu, 22}(\zeta)\left[\mathscr{L}_{21}^{\prime}(\zeta) V_{\mu, 11}-V_{\mu, 22} \mathscr{L}_{21}^{\prime}(\zeta)\right] X_{\mu-1,11}^{-1}(\zeta) d \zeta \\
& +X_{\nu, 22}(z) \mathscr{L}_{21}^{\prime}(z) X_{\nu, 11}^{-1}(z) .
\end{aligned}
$$

Note that all the integrals above exist because the integrands are all asymptotically zero as $\zeta \rightarrow \infty$ along $\gamma_{\mu}$.

Our assumptions on $X_{\nu, j j}$ imply that $\left[A_{j j}(z)\right]$ is a standard example, i.e. of the form

$$
A_{j j}(z)=A_{j, 0} z^{r_{j}-1}+\ldots+A_{j, r_{j}} z^{-1}, r_{j} \quad \text { Poincaré rank of }\left[A_{j j}\right] \text {. }
$$

Inserting this in (5.15) and evaluating $\frac{\zeta^{k}-z^{k}}{\zeta-z}$ for $k=-1, \ldots, r_{j}-1$ shows that the first two sums in (5.15) are again of the standard example type with Poincare rank $r \leqq$ $\max \left(r_{1}, r_{2}\right)-1$. 
To evaluate the third sum in (5.15), we take $R>|z|$ and restrict all the integrals to $|\zeta| \leqq \mathrm{R}$. Using that $X_{m, j j}(\zeta)=X_{0, j j}\left(\zeta e^{-2 \pi i}\right) e^{2 \pi i L_{i j}}$, the third sum equals

$$
\begin{aligned}
& \lim _{R \rightarrow \infty}-\frac{1}{2 \pi i}\left[\sum _ { \mu = 1 } ^ { m - 1 } \left\{\int \frac{1}{\zeta-z} X_{\mu, 22}(\zeta) \mathscr{L}_{21}^{\prime}(\zeta) X_{\mu, 11}^{-1}(\zeta) d \zeta\right.\right.
\end{aligned}
$$

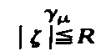

$$
\begin{aligned}
& \left.-\int_{\substack{\gamma_{\mu+1} \\
|\zeta| \geqq R}} \frac{1}{\zeta-z} X_{\mu, 22}(\zeta) \mathscr{L}_{21}^{\prime}(\zeta) X_{\mu, 11}^{-1}(\zeta) d \zeta\right\} \\
& +\int_{\substack{\gamma 0 \\
|\zeta| \leqq R}} \frac{1}{\zeta-z} X_{0,22}(\zeta) e^{2 \pi i L_{22}} \mathscr{L}_{21}^{\prime}\left(\zeta e^{2 \pi i}\right) e^{-2 \pi i L_{11}} X_{0,11}^{-1}(\zeta) d \zeta \\
& \left.-\int_{\gamma_{1}} \frac{1}{\zeta-z} X_{0,22}(\zeta) \mathscr{L}_{21}^{\prime}(\zeta) X_{0,11}^{-1}(\zeta) d \zeta\right]
\end{aligned}
$$

From the definition of $\mathscr{L}_{21}(\zeta)$ we conclude that

$$
\left[\begin{array}{cc}
I & 0 \\
\mathscr{L}_{21}\left(\zeta e^{2 \pi i}\right) & I
\end{array}\right]=\left[\begin{array}{cc}
e^{-2 \pi i L_{11}} & 0 \\
0 & e^{-2 \pi i L_{22}}
\end{array}\right]\left[\begin{array}{cc}
I & 0 \\
\mathscr{L}_{21}(\zeta) & I
\end{array}\right] e^{2 \pi i L},
$$

and by differentiation we obtain, considering the $(2,1)$ block only:

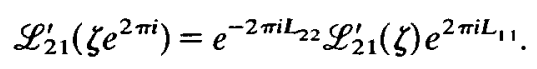

Therefore (5.16) becomes equal to

$$
-\frac{1}{2 \pi i} \lim _{R \rightarrow \infty} \sum_{\mu=0}^{m-1} \int_{\beta_{\mu}(R)} \frac{1}{\zeta-z} X_{\mu, 22}(\zeta) \mathscr{L}_{21}^{\prime}(\zeta) X_{\mu, 11}^{-1}(\zeta) d \zeta
$$

herein $\beta_{\mu}(R)$ denotes the path indicated in Figure 3.

By an appropriate change of the eigenvalues of $M_{\mu_{2}}$ we can ensure the convergence of the above integrals at 0 .

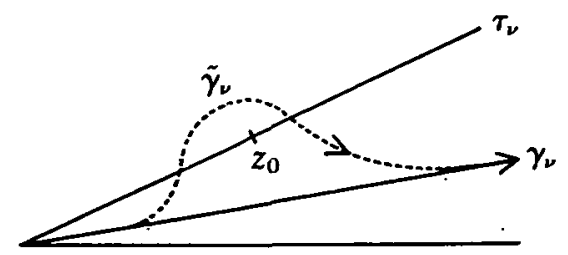

Fig. 3

We now take $R$ fixed and add and subtract integrals taken along the circle $|\zeta|=R$ between $\gamma_{\mu}$ and $\gamma_{\mu+1}$. This leads to integrals along closed curves which are zero except for the path 
that includes $\zeta=z$; in this case we gain a term $-X_{\nu, 22}(z) \mathscr{L}_{21}^{\prime}(z) X_{\nu, 11}^{-1}(z)$ which cancels against the fourth term in (5.15). The leftover terms are

$$
\frac{1}{2 \pi i} \sum_{\mu=0}^{m-1} \int_{\hat{\beta}_{\mu}} \frac{1}{\zeta-z} X_{\mu, 22}(\zeta) \mathscr{L}_{21}^{\prime}(\zeta) X_{\mu, 11}^{-1}(\zeta) d \zeta,
$$

where $\hat{\beta}_{\mu}$ denotes the (positively traced) arc of the circle $|\zeta|=R$ which lies between $\gamma_{\mu}$ and $\gamma_{\mu+1}$. Along $\hat{\beta}_{\mu}$, the integrand in (5.19) can be replaced by a sufficiently large part of its asymptotic expansion, this alters (5.19) by an error term which tends to zero as $R \rightarrow \infty$. The asymptotic expansion, however, is independent of $\mu$ and does not contain exponential terms. Additionally, according to (5.17), it is single valued, hence meromorphic. So we see that (5.19) equals

$$
\frac{1}{2 \pi i} \int_{|\zeta|=R} \frac{1}{\zeta-z} \zeta^{k} P(\zeta) d \zeta
$$

where $k$ is some integer and $P(\zeta)$ a polynomial in $\zeta^{-1}$ which is completely determined by the formal expansions. Using the residue theory we can calculate this integral explicitly, the result does not depend on $R$ and gives the part of $z^{k} P(z)$ which is regular at zero. This finally proves:

If we expand

$$
X_{\mu, 22}(z) \mathscr{L}_{21}^{\prime}(z) X_{\mu, 11}^{-1}(z)=z^{k} F_{0,21}+\ldots+z F_{k-1,21}+F_{k, 21}+O\left(z^{-1}\right) \text { in } S_{\mu},
$$
then

$$
\begin{aligned}
A_{21}(z)= & -\frac{1}{2 \pi i} \sum_{\mu=1}^{m} \int_{\gamma_{\mu}} \frac{1}{\zeta-z}\left(A_{22}(\zeta)-A_{22}(z)\right) T_{\mu, \mu-1,21}(\zeta) d \zeta \\
& +\frac{1}{2 \pi i} \sum_{\mu=1}^{m} \int_{\gamma_{\mu}} \frac{1}{\zeta-z} T_{\mu, \mu-1}(\zeta)\left(A_{11}(\zeta)-A_{11}(z)\right) d \zeta \\
& +z^{k} F_{0,21}+\ldots+z F_{k-1,21}+F_{k, 21},
\end{aligned}
$$

hence $A_{21}(z)$ has at most a first order pole at $z=0$, but its Poincaré rank may exceed the ranks of $\left[A_{j j}\right]$ for $j=1,2$. However, if $L$ is diagonally blocked, i.e. $\mathscr{L}_{21}=0$, this will not happen.

We summarise these results as follows.

Proposition 5. Under the assumptions mentioned for the diagonal blocks $A_{j j}$ (together with their formal fundamental solutions $H_{j j}$, normal solutions $X_{\nu, j}$, and connection matrices $V_{v, j i j}$, and given an "admissible" formal monodromy matrix $L$ and admissible connection matrices $V_{\nu}$ (with the $V_{\nu, j j}$ as diagonal blocks), there are explicitly given "sectorial transformations" $T_{\nu}(z)$, a formal fundamental solution $H(z)$, and normal solutions $X_{\nu}(z)$ having the given invariants and belonging to a differential equation $[A]$, where

$$
A=\left[\begin{array}{cc}
A_{11} & 0 \\
A_{21} & A_{22}
\end{array}\right]
$$

and $A_{21}$ is explicitly given by (5.21), (5.22).

Thus, given reduced meromorphic invariants $z^{L} e^{O}$ and $V_{\nu}$, we can explicitly construct 
a corresponding reduced differential equation $[A]$ provided that the same problem for the diagonal blocks is already solved. The construction is, in principle, the converse of Proposition 4.

\section{Block-reducibility}

In Section 2 we have seen that the conditions for reducibility involve a similarity transformation of the matrices $V_{\nu}$ and $e^{2 \pi i L}$ by means of a diagonally blocked invertible constant matrix $D$. This complicates the application of these conditions, since it is not clear how $D$ should be selected to obtain an optimal type of reducibility. We now will see that this complication can be avoided if we are willing to consider another type of reducibility which is slightly restricted but in some sense quite natural.

Let $[A]$ be given, and let the corresponding $Q$ be arbitrarily ordered such that equal elements appear consecutively, i.e.

$$
Q(z)=\operatorname{diag}\left[q_{1}(z) I_{s_{1}}, \ldots, q_{l}(z) I_{s_{l}}\right]
$$

and $q_{k} \neq q_{j}$ for $k \neq j$ (see $(\mathbf{1}$, Section 1$)$ ). We call a transitive set $\sigma$ consistent with $Q$ if the corresponding incidence matrix $\sigma$ has the property that in the block structure induced by the block structure of $Q(z)$ a block of $\sigma$ contains all ones or all zeros as its elements. In this section we will consider only such transitive sets $\sigma$ which are consistent with $Q$ for some suitable ordering of $Q$ satisfying (6.1).

A matrix is called block-reduced of type $\sigma$ if it is reduced of type $\sigma$ for a $\sigma$ which is consistent with some $Q$ satisfying (6.1); it is said to be block-reduced of type $\sigma^{+}$if it is the sum of the identity matrix and a matrix which is block-reduced of type $\sigma$.

A meromorphic differential equation $[A]$ is said to be block-reduced of type $\sigma$, if it has a formal fundamental solution $H=\Psi e^{Q}$ with $Q$ satisfying (6.1), $H$ being block-reduced of type $\sigma^{+}$and $\sigma$ is consistent with $Q$. Note that this definition differs from the corresponding one in Section 2; in fact [A] being block-reduced of type $\sigma$ implies that the coefficient matrix $A(z)$ is block-reduced of type $\sigma$ whereas the latter implies the existence of an $\tilde{H}=\tilde{\Psi} e^{\bar{Q}}$ which is block-reduced of type $\sigma^{+}$, however $\tilde{Q}$ may possibly not satisfy (6.1), or $\sigma$ may not be consistent with $\tilde{Q}$.

We call [A] $v$-meromorphically block-reducible of type $\sigma$ if $[A]$ is $v$-meromorphically equivalent to, say, $[\tilde{A}]$ which is block-reduced of type $\sigma$.

We again call two sets $\sigma$ and $\tilde{\sigma}$ equivalent, if the corresponding incidence matrices are related by

$$
\tilde{\sigma}=R^{-1} \sigma R \text {, }
$$

where $R$ is a block permutation matrix, i.e. $\tilde{Q}=R^{-1} Q R$ again satisfies (6.1).

With these notions we can establish an analogue to the results of Section 2. Let again a formal fundamental solution $H=\Psi e^{Q}$ of $[A]$ with $Q$ satisfying (6.1) being selected, and let $e^{2 \pi i L}$ and $\left(V_{\nu}\right)$ denote the corresponding formal circuit matrix resp. the normalised connection system.

Theorem 3. A meromorphic differential equation $[A]$ is v-meromophically blockreducible of type $\sigma$ iff there exists a set $\sigma$ which is consistent with $Q$ and equivalent with $\sigma$ such that 
are block-reduced of type $\tilde{\sigma}^{+}$.

$$
e^{Q}, e^{2 v \pi i L}, \text { and } V_{\nu} \text { forall } \nu
$$

Remark. We see that in condition (6.3) no diagonally blocked D appears. This makes it easy to check whether (6.3) holds. In case that all the elements of $Q$ are distinct, i.e. all $s_{j}=1$ for $j=1, \ldots, l$ then these new notions completely coincide with the ones of Section 2 and (6.3) can be directly seen to be equivalent to the corresponding condition of Theorem 1 resp. its Corollary.

A proof of Theorem 3 follows easily by an inspection of the proof of Theorem 1 and can be omitted here.

If we want to block-reduce a given [ $A$ ] as far as possible disregarding the roots which may come into the new equation, we can see that there always exists a best-possible $\sigma$ in the sense that every other possible type of block-reducibility for $[A]$ contains a type which is equivalent to $\sigma$. To this extent, the formal solution $H$ of $[A]$ may be taken to be meromorphically normalised (see $(\mathbf{1}$, Sections 1,2$)$ ). Then

$$
e^{2 \pi i L}=D R
$$

where $R$ is a direct sum of superblocks, each of which has the form

$$
\left[\begin{array}{lllll}
0 & \cdot & \cdot & \cdot & I_{s} \\
I_{s} & \cdot & & & \cdot \\
\cdot & & \cdot & & \cdot \\
\cdot & & & \cdot & . \\
0 & \cdot & \cdot & I_{s} & 0
\end{array}\right],
$$

and $D$ is diagonally blocked, constant and invertible and commutes with $R$.

If we take $v$ to be a multiple of the order of $R$ (i.e. of the smallest integer $\mu$ such that $\left.R^{\mu}=I\right)$, then $e^{2 v \pi i L}=D^{\nu}$ is diagonally blocked. Hence if $\sigma$ is the smallest set of block-indices such that all $V_{\nu}$ are block-reduced of type $\sigma^{+}$, the condition that $e^{Q}$ and $e^{2 v \pi i L}$ are also block-reduced of type $\sigma^{+}$can be made true by simply adding those diagonal positions to $\sigma$ where the corresponding blocks of $e^{Q}$ or $D^{v}$ differ from $I$. This additional set can be minimised by taking $v$ also as a multiple of the order of those blocks of $D$ which are "roots of unity", i.e. which have an integer power equalling $I$. Other choices of $v$ do not lead to a smaller set $\sigma$, since the off-diagonal positions have to contain at least all those index pairs where at least one $V_{\nu}$ has a non-zero block, and the additional set of diagonal positions is minimal, since a diagonal block of $e^{2 v \pi i L}=D^{\nu} R^{\nu}$ is either 0 or the $v$ th power of the corresponding diagonal block of $D$.

Thus, apart from locating the non-zero blocks in $Q$ and the blocks in $D$ which are not roots of unity, we have to find the smallest $\sigma$ such that all $V_{\nu}$ are block-reduced of type $\sigma^{+}$.

This can be done as follows, using only $V_{1}, \ldots, V_{m}$ :

(i) Find the smallest set $\sigma_{1}$ such that $V_{1}, \ldots, V_{m}$ are block-reduced of type $\sigma_{1}^{+}$.

(ii) If a set $\sigma_{j}$ is defined, take

$$
\sigma_{j+1}=\sigma_{j} \cup R^{-i} \sigma_{1} R^{i} \quad \text { for } \quad j=1,2, \ldots
$$

Follow this procedure until for the first time $\sigma_{k_{0}}=\sigma_{k_{0}+1}$.

If $\sigma$ is defined to be the transitive closure of $\sigma_{i_{0}}$, then $\sigma$ is the smallest transitive set such that all $V_{\nu}$ are block-reduced of type $\sigma^{+}$. 
To see this, observe that $\sigma_{\mu}=\sigma_{\mu+1}$, where $\mu$ is the order of $R$, hence $j_{0} \leqq \mu$ exists, and

$$
\begin{aligned}
\sigma_{j+1} & =\sigma_{1} \cup R^{-1} \sigma_{1} R \cup \ldots \cup R^{-j} \sigma_{1} R^{j} \\
& =\sigma_{1} \cup R^{-1} \sigma_{j} R, \text { for } j \geqq 1 .
\end{aligned}
$$

Hence $R^{-1} \sigma_{j, 0} R \subseteq \sigma_{j, 0}$, and consequently $R^{-1} \sigma R \subseteq \sigma$. In view of $R^{-1}=R^{\mu-1}$ we see by iteration that $R^{-\bar{k}} \sigma R^{k} \subseteq \sigma$ for all integers $k$. Since $V_{\nu}$ are block-reduced of type $\sigma^{+}$for $\nu=1, \ldots, m$, we have that

$$
V_{\nu+k m}=R^{-k} D^{-k} V_{\nu} D^{k} R^{k}
$$

is block-reduced of type $\sigma^{+}$for all integers $k$ (note that $D^{-k}\left(I-V_{\nu}\right) D^{k}$ has the same zero/non-zero block structure as $I-V_{\nu}$ ). The minimality of $\sigma$ is clear by construction and (6.7).

\section{REFERENCES}

$(1,2)$ W. BALSER, W. B. JuRKAT, and D. A. LuTZ, A general theory of invariants for meromorphic differential equations, Parts I \& II, Funk. Ekvac. (to appear).

(3) W. BALSER, W. B. JURKAT and D. A. LUTZ, Birkhoff invariants and Stokes' multipliers for meromorphic linear differential equations, J. Math. Anal. Appl. 71 (1979), 48-94.

(4) F. R. GANTMACHER, Theory of Matrices, vol. I \& II (Chelsea, New York, 1959).

(5) L. M. HALL, The mumber of analytic solutions of a singular differential system, preprint.

(6) W. B. JURKAT, Meromorphe Differentialgleichungen (Lecture Notes in Mathematics No 637, Springer Verlag, Berlin, Heidelberg, New York, 1978).

(7) W. B. Jurkat, D. A. LuTz and A. PeyerimhofF, Birkhoff invariants and effective calculations for meromorphic linear differential equations, Part I, J. Math. "Anal. Appl. 53 (1976), 438-470; Part II, Houston J. Math. 2 (1976), 207-238.

(8) B. Malgrange, Sur les points singuliers des équations differentielles, L'Enseignement Mathématiques, vol. XX, 1-2 (1974), 147-176.

UNIVERSITÄT ULM
D. 7900 ULM
WEST GERMANY

UNIVERSITY OF WISCONSIN-MILWAUKEE

MILWAUKEE

WISCONSIN 53261
SYRACUSE UNIVERSITY

NEW YORK 13210 